\title{
MedienPädagogik
}

Zeitschrift für Theorie und Praxis der Medienbildung

Themenheft Nr. 38: Aneignung politischer Information in Social Media und Internet. Forschungslage und medienpädagogische Implikationen Herausgegeben von Kai Hugger, Kai Kaspar und Lars Gräßer

\section{Politische Bildung für Jugendliche in oder mit digitalen Medien?}

\section{Medienpädagogische Reflexionen der Strukturbedingungen von YouTube}

Julian Ernst und Josephine B. Schmitt

\section{Zusammenfassung}

Webvideoreihen, moderierte Kommentarspalten und Umfragetools - politische Bildung wird längst nicht mehr nur in pädagogischen Settings beansprucht, wie etwa im Schulunterricht, sondern auch in Angeboten digitaler Medien, etwa auf Plattformen wie YouTube. Doch kann politische Bildung in digitalen Medien gelingen? Oder ist eine politische Bildung, die ihren Anspruch ernst nimmt, nicht eher eine politische Bildung mit digitalen Medien, d.h. weiterhin auf pädagogische face-to-face Kommunikationen angewiesen? Entlang zweier zentraler Strukturbedingungen der Plattform YouTube - Nutzerinnen- und Nutzerkommentare und Algorithmen - möchte der vorliegende Beitrag diese Fragen näher diskutieren.

Civic Education for Youths in or by means of Digital Media? Media-pedagogical reflections on structural characteristics of YouTube.

\begin{abstract}
Webvideos, moderated comment sections and survey tools - not only traditional pedagogical settings such as school claim to offer civic education, but also different kinds of digital media formats, for example, on platforms like YouTube. But, can civic education only delivered by digital media be successful? Or, is an offline - or even faceto-face - pedagogical setting still an important boundary condition for a succeeding civic education? In other words, should we regard digital media rather as a tool than as an environment for civic education? In the present paper, we want to discuss these questions alongside two significant structural characteristics of the online platform YouTube - user comments and algorithms.
\end{abstract}




\section{Einleitung}

«Medien sind gegenständliche Werkzeuge, mit denen Lernende in Lernsituationen umgehen und die als Arbeitsmittel und Informationsträger in didaktischer Absicht ausgewählt, gestaltet und verwendet werden, um Hilfen zum Lernen zu bieten. Zu einem erheblichen Teil handelt es sich in der Praxis zugleich um eine didaktisch begründete Auswahl solcher Medien, in denen zugleich die politische Diskussion stattfindet.» (Sander 2005, 30)

In dieser Weise wurden die Funktionen von Medien in der politischen Bildung ${ }^{1}$ noch vor einigen Jahren beschrieben. Aus dem kurzen Zitat spricht ein klassisches Verständnis von Medien und Unterricht bzw. pädagogischer Situation: Medien sind Pädagoginnen und Pädagogen als «Werkzeuge» dienlich und bieten «Informationen» für den (Politik-)Unterricht, dokumentieren bzw. repräsentieren «die politische Diskussion».

Seither haben sich die Medienlandschaft und der Stellenwert von Medien im Alltag von Menschen dynamisch und radikal verändert. Insbesondere digitale Medi$e^{2}{ }^{2}$ sind gerade für jugendliche Nutzerinnen und Nutzer mehr als nur «Werkzeuge», die den Alltag ergänzen oder in denen sich die Welt offenbart. Digitale Medien sind längst tief in die Lebenswelt Jugendlicher vorgedrungen, sie sind in dieser nicht nur relevant, sondern strukturieren die Relevanzsetzungen in dieser: Sie geben einen Rahmen vor, was von der Welt, in welcher Art und Weise diese wahrgenommen wird (Leven und Utzmann 2020; Krotz 2007).

Diese Veränderung lässt sich als Mediatisierung oder auch Digitalisierung der Lebenswelt beschreiben - und sie wirkt auch in die pädagogische Sphäre zurück. Diese Entwicklung könnte Pädagoginnen und Pädagogen eigentlich positiv stimmen: Dank der vielfältigen Möglichkeiten der Produktion und Bereitstellung von Inhalten im Netz können Akteurinnen und Akteure der schulischen und ausserschulischen Bildungsarbeit auf unzählige (multimedial aufbereitete) Inhalte, Materialien, Anregungen etc. zugreifen.

Gleichzeitig bringen digitale Medien jedoch neben den möglichen positiven Eigenschaften und Potentialen eine Vielzahl an Herausforderungen mit sich. So kann die Vielfalt und Diversität von Inhalten etwa zu Stress und Überforderung bei

1 Unter «politischer Bildung» sollen im vorliegenden Artikel pädagogische Formate wie beispielsweise das Schulfach Gesellschaftslehre oder auch ausserschulische Angebote verstanden werden, die zum Ziel haben, verschiedene Gruppen in ihrer Mündigkeit zu fördern, u.a. bezogen auf die Fähigkeit, an politischen Diskussionen und einer demokratischen Gesellschaft selbstbestimmt und kritisch teilhaben zu können (vgl. z.B. Reheis 2016, 15 f.). Politische Bildung wird insofern als möglicher Anlass von Bildungsprozessen verstanden, d.h. als möglicher Anlass der Veränderung von Selbst- und Weltbezügen (vgl. Jörissen und Marotzki 2009, 23) - hierzu weiter in Abschnitt 2.

2 Mit digitalen Medien bezeichnen wir im vorliegenden Artikel sowohl Bedeutungsentitäten bzw. mediale Artikulationen wie Videos, Kommentare o.ä. (Jörissen und Marotzki 2009, 38 f.) als auch Ausprägungen einer «Kultur der Digitalität» im Sinne von Plattformen wie etwa YouTube und ihren gegenüber analogen Medien charakteristischen Funktionsweisen (Stalder 2016, 12 f.). 
Nutzerinnen und Nutzern führen (Schmitt, Debbelt, und Schneider 2017; Tarafdar et al. 2019). Darüber hinaus ist das Internet bevorzugtes Mittel für die Verbreitung von problematischen Inhalten, wie etwa Hate Speech, sogenannten «Fake News», Cybermobbing etc. (siehe z.B. Schmitt et al. 2017; Wachs 2017). Insbesondere soziale Medien werden nicht nur als Kanäle für die Bereitstellung, Kommunikation und Diskussion von Bildungsinhalten verwendet, sondern bieten Raum für die Kommunikation von und mit problematischen Akteurinnen und Akteuren (s. z.B. SchattoEckrodt et al. 2019). Eine weitere zentrale Herausforderung vieler digitaler Medien sind automatisierte Algorithmen, die im Hintergrund vieler Plattformen die Relevanz und Sichtbarkeit von Inhalten - und damit möglicherweise auch Meinungen - beeinflussen (Klinger und Svenson 2018; Wallace 2018)

Angesichts der zuvor skizzierten Herausforderungen digitaler Medien stellen sich folgende Fragen für digital verfügbare bzw. vermittelte Bildungsangebote: Kann politische Bildung in digitalen Medien gelingen? Oder ist politische Bildung, die Bildungsprozesse im Sinne der Veränderung "von Welt- und auch Selbstverhältnissen» (Jörissen und Marotzki 2009, 23) anzustossen beansprucht, weiterhin auf pädagogische Kommunikationen face-to-face angewiesen? Wie kann politische Bildung mit digitalen Medien im Zeichen zunehmender Digitalisierung und ihrer Herausforderungen - insbesondere die der algorithmischen Verschränkung von Inhalten und der sogenannten Hassrede - didaktisch gestaltet werden? Der Beitrag möchte diese Fragen vor dem Hintergrund (empirischer) kommunikations- und erziehungswissenschaftlicher Untersuchungen sowie medienpsychologischer Studien näher diskutieren. Ausgehend davon werden Implikationen für die medienpädagogische Praxis sowie für die Lehrpersonenbildung abgeleitet.

Besonderes Augenmerk liegt auf Angeboten der politischen Bildung, die für die (primäre) Radikalisierungsprävention entwickelt worden sind bzw. diese zum Ziel haben. Diese Auswahl spiegelt damit nur eine mögliche Facette politischer Bildung wider. Sie eignet sich jedoch besonders, um Herausforderungen digitaler Medien für die politische Bildung herauszuarbeiten.

Im Fokus steht die Plattform YouTube - ein soziales Medium ${ }^{3}$, das sich gerade unter jugendlichen Mediennutzerinnen und -nutzern grosser Beliebtheit erfreut: einerseits als Unterhaltungsmedium (mpfs 2019), andererseits auch als «digitaler Lernort» (Rat für Kulturelle Bildung 2019). Diese Beliebtheit wollen sich zunehmend auch Bildungsinstitutionen zu Nutze machen, u.a. da die Plattform eine kostengünstige und schnelle Verbreitung von Inhalten an ein disperses Publikum verspricht (Statista 2020). Die Diskussion der o.g. Forschungsfragen soll dabei entlang zweier zentraler Strukturbedingungen der Plattform erfolgen, welche die Auswahl, Wahrnehmung

3 Digitale Plattformen wie YouTube, Instagram o.ä., die die Möglichkeit zur Interaktion mit anderen Nutzerinnen und Nutzern und die Publikation selbsterstellter Inhalte bieten, werden auch als soziale Medien bezeichnet. 
und Bewertung von Bildungsangeboten durch Nutzende massgeblich beeinflussen können: Algorithmen und Nutzerinnen- und Nutzerkommentare.

In einem ersten Schritt werden dazu die Herausforderungen, die das Internet an die Nutzerinnen und Nutzer und die politische Bildung stellt, aus einer allgemeinen medienpädagogischen, kommunikationswissenschaftlichen und medienpsychologischen Perspektive eingehender erörtert. Darauffolgend wird der Fokus auf eine häufige Zielgruppe politischer Bildungsangebote verengt: jugendliche Nutzerinnen und Nutzer.

\section{Strukturbedingungen digitaler Medien: Potenziale und Hürden für Bildungsprozesse}

Wie oben bereits angedeutet, erscheint es in der Sache zunehmend inadäquat (digitale) Medien ausschliesslich als Werkzeuge oder schlicht als Raum anzusehen. Im Verständnis einer in den letzten Jahren begrifflich wie konzeptionell prominenter werdenden Medienbildung sind Medien vielmehr ihrerseits als Gegenstände und zugleich, ob ihrer Medialität, als Strukturbedingungen ${ }^{4}$ von Bildungsprozessen anzusehen und pädagogisch zu adressieren (z.B. Bettinger 2018; Jörissen und Marotzki 2009).

Benjamin Jörissen und Winfried Marotzki (2009) stellen in ihrem theoretischen Ansatz zur Medienbildung die «strukturalen» Aspekte ausgewählter «medialer Artikulationsformen» heraus, Bildung zu initiieren. Bildung wird dabei als Prozess der Veränderung «von Welt- und auch Selbstverhältnissen» bzw. des Erwerbs von «Orientierungswissen» verstanden, welcher vom Wissenserwerb im Sinne des Erschliessens von Inhalten zu unterscheiden ist (vgl. ebd., 23, 27 f.). Nach Jörissen und Marotzki lassen sich anhand der unterschiedlichen Erscheinung- und Artikulationsweisen von Medien bestimmte Strukturen dieser identifizieren, welche «Bildungspotentiale» bergen, d.h. in ihrer je spezifischen Strukturiertheit potentiell Bildungsprozesse ermöglichen (vgl. ebd., 25, 30). So nennen sie etwa die Besonderheit verschiedener Plattformen, einen Avatar anzulegen, als Möglichkeit, in Form eines selbstgewählten Charakters in eine Welt einzutauchen, einen Perspektivwechsel zu vollziehen und so u.U. Bildungsprozesse initiieren zu können (vgl. ebd., 209 f.).

Was in den Überlegungen jedoch nur im Ansatz zum Ausdruck kommt (vgl. ebd., 30), sind die für (post-)moderne Gesellschaften und die dort einhergehenden technisch-mediale Entwicklungen charakteristischen Momente, welche den mündigen, kritischen und selbst-denkenden - in diesem Sinne sich bildenden - Subjekten entgegenwirken (grundlegend beschrieben werden diese Momente etwa bei Horkheimer

4 Angelehnt soll sich mit dem Begriff an die von Jörissen und Marotzki genannten «strukturalen Bedingungen» (vgl. Jörissen und Marotzki 2009, 40, kursiv i.O.). 
und Adorno 1969). ${ }^{5}$ In anderen Worten formuliert: dass (gerade digitale) Medien nicht nur strukturelle Potenziale für Bildungsprozesse bieten, sondern auch Strukturen, die diese verhindern können. Dabei soll an dieser Stelle weder einer «bewahrpädagogischen» Argumentationsweise noch einer grundsätzlichen Technikskepsis o.ä. Vorschub geleistet werden. Vielmehr soll der dialektische Charakter der Möglichkeit von Bildungsprozessen in einer digitalisierten Gesellschaft unterstrichen werden.

Es lassen sich an dieser Stelle eine ganze Reihe an Phänomenen im Kontext digitaler Medien aufzählen, denen eine Wirkung unterstellt werden kann, die (demokratisch wünschenswerte) Bildungsprozesse beeinträchtigen oder gar verhindern kann. Ein Beispiel wäre Hate Speech ${ }^{6}$, insbesondere Hasskommentare und extremistische Propagandavideos. Unlängst sind mehrere Studien zu dem Schluss gekommen, dass die Begegnung mit gruppenbezogenen Abwertungen eine alltägliche Facette des Medienhandelns Jugendlicher darstellt (Reinemann et al. 2019; mpfs 2019; LfM NRW 2016; Rieger et al. 2013). Auch sogenannte «Fake News», die derzeit im Zusammenhang mit autoritären Einstellungen in (post-)modernen Gesellschaften diskutiert werden, können hier genannt werden (z.B. Milbradt 2018; Stalder 2017).

Ganz grundlegend kann die schiere Masse möglicher Informations- und Unterhaltungsangebote in digitalen Medien als strukturelle Herausforderung angeführt werden. Ein erhöhter «Selektionsdruck» (Busch 2017, 55) - medienbildungstheoretisch könnte man von einer Anforderung an das Orientierungswissen sprechen setzt viele Menschen auch psychisch unter Druck (s. z.B. Schmitt et al. 2017). Flankiert wird dies durch spezifische Funktionsweisen bzw. Strukturen von Plattformen, wie etwa Algorithmen und Nutzerinnen- und Nutzerkommentare, die Vorselektionen, Verknüpfungen oder auch erste Interpretationen von Medienangeboten anbieten.

Die skizzierten Entwicklungen digitaler Medien lassen auch das Praxisfeld der politischen Bildung nicht unberührt. Im Gegenteil: Digitale Medien und Phänomene der Digitalisierung werden als ein (neuer?) wichtiger Gegenstandsbereich politischer Bildung diskutiert (siehe z.B. Kenner und Lange 2020; Gapski, Oberle, und Staufer 2017; Pöttinger, Kalwar, Fries 2016; Schaumburg 2011; Moser 2011). Insbesondere gewinnen das Internet im Allgemeinen und die Verwendung von sozialen Medien im Speziellen an Bedeutung. Neben (interaktiven) Informationsangeboten geraten Plattformen wie YouTube oder Instagram als Raum politischer Teilhabe in den Fokus.

Von verschiedenen staatlichen und zivilgesellschaftlichen Institutionen und Akteurinnen und Akteuren werden etwa Instagram, Facebook und YouTube, explizit für die politische Bildung genutzt: So veröffentlicht die Bundeszentrale für politische Bildung (bpb) seit einigen Jahren Webvideoreihen zu unterschiedlichsten Themen bei YouTube (z.B. «Begriffswelten Islam», bpb 2015), begleitet von entsprechend

5 Ein Grund hierfür könnte das Zurücktreten des handelnden Subjekts zugunsten medialer Artikulationen im Ansatz von Jörissen und Marotzki sein (s. auch Hugger und Cwielong 2009 sowie Bettinger 2018, 87 f.).

6 Gegenüber schlichten Beleidigungen und Unhöflichkeiten soll Hate Speech durch ihre Referenz auf soziale Gruppen entlang gesellschaftlicher Machtverhältnisse unterschieden werden (siehe z.B. Butler 2016). 
moderierten Kommentarspalten. Mittlerweile zieht auch der nordrhein-westfälische Verfassungsschutz mit einer Webvideo-Reihe nach, die sich mithilfe von Satire und Aufklärung mit dem Thema Islamismus befasst. ${ }^{7}$ Weiterhin sind funk, das digitale Jugendangebote von ARD und ZDF, oder auch die datteltäter, ein Kanal muslimischer YouTuberinnen und YouTuber ${ }^{8}$, als Reichweiten-starke Akteure auf dem Gebiet politischer (Bildungs- bzw. Informations-) Angebote über YouTube zu nennen. ${ }^{9}$

Auch die App Instagram wird zunehmend als Kanal für die politische Bildung entdeckt (siehe das von der bpb geförderte Projekt «Say My Name», bpb 2019a oder die Story-Serie «Throwback89» der Tagesschau zum Mauerfall, tagesschau 2019). Aber auch Tools wie der «Wahlomat» (bpb 2019b) oder auch verschiedene Quizformate stehen online zur Verfügung (z.B. handysektor 2019). Primäre Zielgruppe dieser Angebote sind in der Regel die Jugendliche, die in ihrem alltäglichen Medienhandeln durch die Bildungsangebote erreicht werden sollen.

\section{Digitale Medien: Jugendliches Medienhandeln und politische Bildung zwischen} Unterhaltung, Information und Manipulation

Das Internet ist selbstverständlicher Bestandteil jugendlichen Medienhandelns. Das schlägt sich nicht nur in der Menge der internetfähigen Geräte in deutschen Haushalten nieder, sondern auch darin, dass die Nutzung von Smartphones und anderen internetfähigen Geräten zu den wichtigsten Freizeitbeschäftigungen Jugendlicher zählt (mpfs 2019). Unterhaltung und Kommunikation mithilfe sozialer Medien sind dabei die wichtigsten Nutzungsmotive. Als Angebot steht insbesondere die Videoplattform YouTube im Zentrum der Aufmerksamkeit (mpfs 2019; Calmbach et al. 2016): Sie wird nicht nur von ca. zwei Dritteln der Jugendlichen als Lieblingsonlineangebot genannt, sondern zählt bei einem Grossteil gar als bedeutsamstes OnlineUnterhaltungsangebot.

Die Bedeutung sozialer Online-Angebote rührt insbesondere daher, dass sie zahlreiche Anknüpfungspunkte für die Bewältigung von Entwicklungsaufgaben im Jugendalter bieten. So ermöglichen sie die Erschliessung gesellschaftlicher Handlungsspielräume, Autonomie und Zugehörigkeit (Hugger und Braun 2015; Hurrelmann, 2012; Süss und Hipeli 2010). Darüber hinaus werden soziale Medien insbesondere von Jugendlichen und jungen Erwachsenen aufgrund der vermeintlichen Einfachheit, Verständlichkeit und Aktualität der Inhalte als wichtige Informationsquellen geschätzt, wobei sie zuweilen den Informationsgehalt und die Glaubwürdigkeit der dargebotenen Inhalten in Frage stellen (Wolfert und Leven 2020; mpfs 2019; Schmitt 2014).

7 https://www.youtube.com/channel/UCCkTQUPNZjw8VFIZWB2CMfg/about (Stand 11. Juli 2020).

8 Datteltäter ist zwar ein eigener YouTube-Kanal, ist aber ebenfalls seit 2016 ein Angebot von funk.

9 https://www.youtube.com/channel/UCOgPGtSnFR6GM-AkzCnxqMQ (Stand 14. Juli 2020). 
Zwei Strukturbedingungen digitaler Medien, denen ein besonderer Einfluss auf das Medienhandeln (nicht nur) Jugendlicher zugeschrieben werden kann, sind Algorithmen bzw. algorithmisch generierte Empfehlungen sowie die Funktion der Nutzerinnen- und Nutzerkommentare. Diese beiden Strukturbedingungen bilden zentrale Aspekte vieler sozialer Medien ab: einerseits eine plattformseitige «Manipulation» der Sichtbarkeit und Relevanzbestimmung von Online-Inhalten (Algorithmen), andererseits eine Nutzerinnen- und Nutzerseitige Rahmung und Diskussion von Inhalten, die ihrerseits die Bewertung und Selektion von Online-Inhalten der politischen Bildung beeinflussen können. Auf die digitalen Herausforderungen für die politische Bildung, die sich aus den beiden Strukturbedingungen ergeben, soll folgend näher eingegangen werden.

\subsection{Digitale Herausforderung für die politische Bildung I: Algorithmen}

Die Informationsmenge und -vielfalt im Internet bzw. das Nebeneinander von nutzergenerierten und redaktionell erstellten Inhalten stellen Nutzerinnen und Nutzer wie Produzentinnen und Produzenten politischer Bildungsangebote vor nicht unwesentliche Herausforderungen (Eder, Mikat, und Tillmann 2017). Auf zahlreichen OnlinePlattformen beeinflussen automatisierte Algorithmen im Hintergrund die Auswahl und Nutzung von Angeboten (Lazer 2015). Bei YouTube etwa «empfiehlt» ein Algorithmus auf der Grundlage von Stichworten, bisheriger eigener YouTube-Nutzung bzw. der Nutzung vermeintlich ähnlicher Nutzerinnen und Nutzer, und dem Wissen über den Ort, an dem sich der/die Nutzende befindet, Videos, die ebenfalls interessieren könnten (s. Abbildung 1). Die Motive des Konzerns sind dabei nicht uneigennützig. Es geht darum, die Nutzung der Plattform so angenehm wie möglich zu machen, damit Nutzende möglichst viel Zeit dort verbringen, viele Videos (beginnen zu) schauen, was wiederum finanziellen Gewinn für die Plattform bedeutet.

Algorithmen können nicht nur die Art, Diversität und Relevanz von Informationen beeinflussen, sondern indirekt auch das gesellschaftliche Klima und die öffentliche Meinung (Bakshy et al. 2016; Helberger, Karppinen, und D’Acunto 2016; Saurwein, Just, und Latzer 2015). 


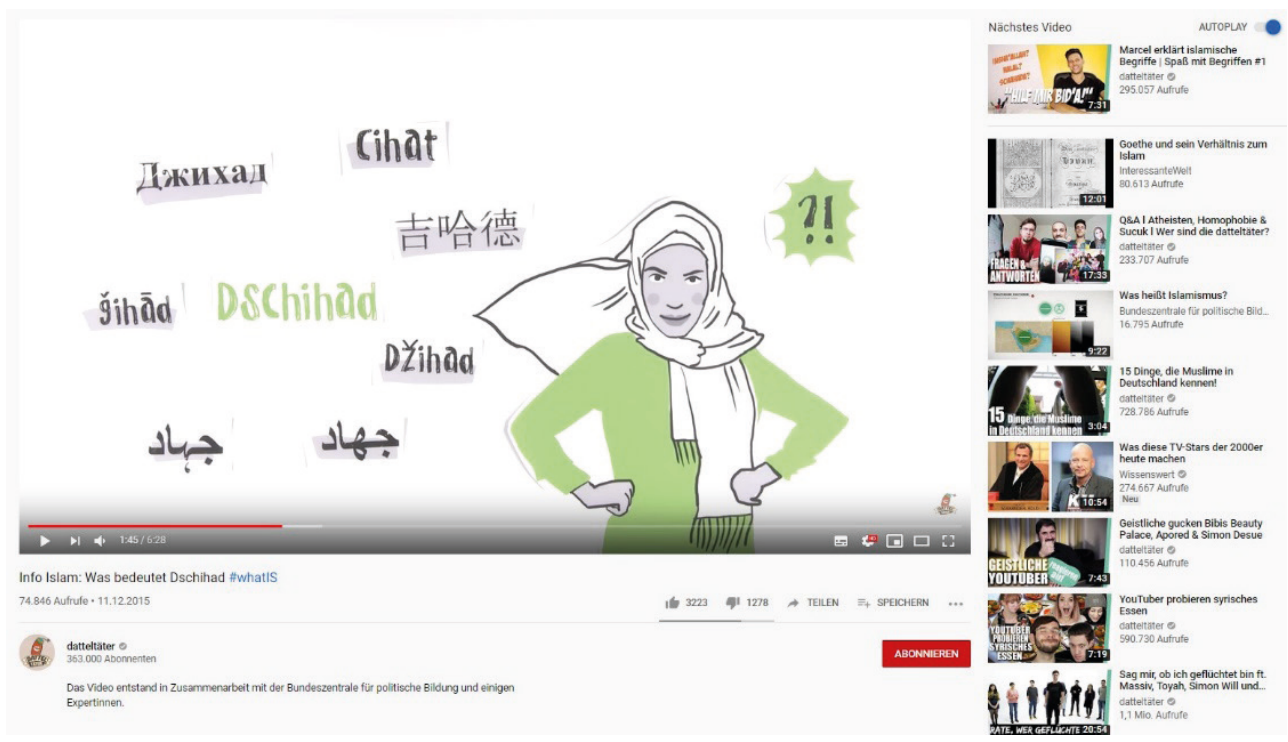

Abb. 1.: Beispiel eines YouTube-Video aus Rezipientinnen- und Rezipientenperspektive mit algorithmisch generierten «Empfehlungen» weiterer Videos am rechten Rand des Interfaces.

Im Rahmen einer Untersuchung der algorithmischen Vernetzung von Online-Bildungsangeboten der bpb im Hinblick auf die Aufklärung über zentrale Begriffe des Islam (bpb 2015) konnte gezeigt werden, inwiefern eine solche algorithmische Verknüpfung im Internet problematische Folgen für den Anspruch politischer Bildung haben kann. Ausgehend von den acht Webvideos der Webvideoreihe "Begriffswelten Islam» wurde ein Netzwerk derjenigen Videos gebildet, die über die Empfehlungsliste von YouTube als «relevant» auf erster und zweiter Ebene eingestuft wurden. Ausgehend davon wurde das ca. 12.000 Videos umfassende Netzwerk rund um die Videos der Webvideoreihe einer Inhaltsanalyse unterzogen (vgl. Schmitt et al. 2018, 8).

Hierbei fanden die Autorinnen und Autoren, dass maximal zwei Klicks in der Empfehlungsleiste von YouTube notwendig sind, um von einem Bildungsangebot etwa über das Begriffspaar «halal/haram» zu einem Angebot eines extremistischen Akteurs zu gelangen (Schmitt et al. 2018). Das ist insofern problematisch, als dass die durch den YouTube-Algorithmus zur Verfügung gestellten «Empfehlungen» insbesondere von jüngeren Mediennutzerinnen und -nutzern als soziale Hinweisreize ausgelegt werden können, d.h. dass jüngere Nutzerinnen und Nutzer diesen potentiell vertrauen und folgen. Dies ist vor dem Hintergrund der Befunde über die enge Vernetzung von Bildungsangeboten und extremistischen Botschaften eine besondere und herausfordernde Situation für Bildungsakteurinnen und -akteure in sozialen Medien. 
Algorithmische Empfehlungen vermeiden damit tendenziell nicht nur die Irritation bereits verfügbarer Deutungsmuster von Subjekten (vgl. Waldenfels 2016; ferner: Jörissen und Marotzki 2009, 19 f.). Sie können dadurch letztlich (Rezeptions-) Situationen mit dem besonderen Potential vorbeugen, Bildungsprozesse anzustossen (vgl. Jörisssen und Marotzki 2009). Sie können darüber hinaus gar zu Inhalten führen, die auf eine Verfestigung ideologischer Weltdeutung abzielen (Baaken und Meyer 2019).

\subsection{Digitale Herausforderung für die politische Bildung II: Nutzerinnen- und Nutzerkommentare}

Neben Algorithmen stellen Nutzerinnen- und Nutzerkommentare unter Online-Inhalten wie Artikeln und Videos, die längst als Standard-Feature sozialer Medien bezeichnet werden können (s. Abb. 2; Ziegele 2016), die politische Bildung im Internet vor Probleme. So finden sich neben konstruktiven oder vergleichsweise banalen Kommentaren und Unterhaltungen häufig auch Kommentare, welche die Herabwürdigung und Verunglimpfung von Personen oder gesellschaftlichen Gruppen beinhalten, i.e. Hate Speech (siehe z.B. Ziegele et al. 2018).

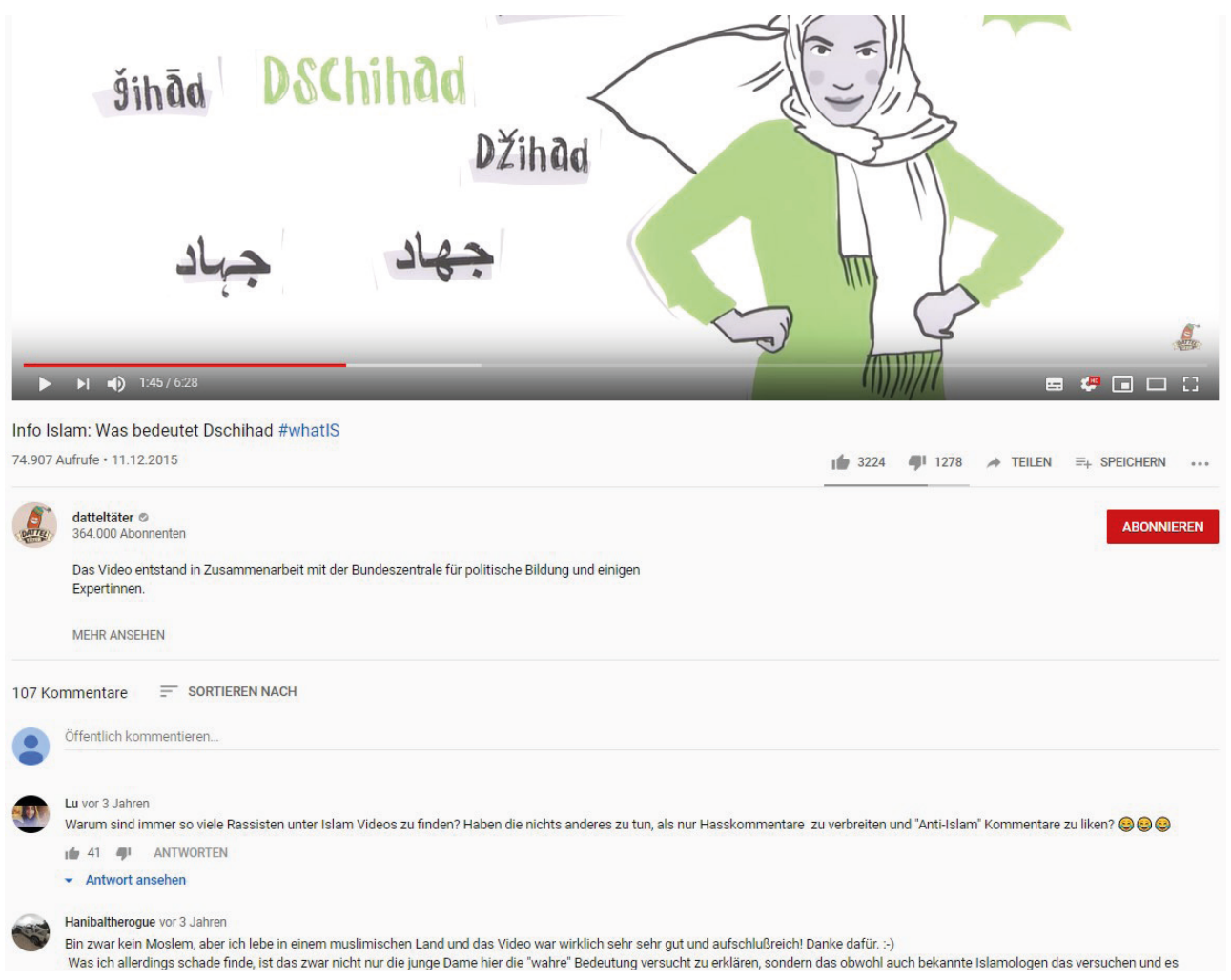

Abb. 2.: Beispiel eines YouTube-Video aus Rezipientinnen- und Rezipientenperspektive mit Nutzerinnen- und Nutzerkommentaren in der unteren Bildhälfte. 
Eine Untersuchung der Landesanstalt für Medien Nordrhein-Westfalen zeigt etwa, dass 54\% der befragten Jugendlichen «sehr häufig» und «häufig» auf Hassbotschaften online treffen. Immerhin rund 37\% machen Angaben, «weniger häufig» auf entsprechende Inhalte zu treffen und nur $8 \%$ geben an, «noch nie» auf Hass online gestossen zu sein (LFM NRW 2016). Dies deckt sich mit Befunden der aktuelle ShellJugendstudie, gemäss der über $50 \%$ der Jugendlichen der Meinung sind, dass in sozialen Medien häufig "Gehässigkeiten verbreitet werden, die andere blossstellen oder verletzen» (Wolfert und Leven 2020, 236). Aber auch für viele Erwachsene gehören hasserfüllte Äusserungen mittlerweile zum Online-Alltag (Geschke, Klaßen, Quent, und Richter 2019).

Dass Hasskommentare längst zum Alltag des Medienhandelns geworden sind, ruft doch gerade Bestrebungen auf den Plan, die sich dezidiert gegen Hate Speech und für Menschenrechte, die freiheitlich demokratische Grundordnung und weitere demokratietheoretisch wünschenswerte Weltdeutungen einzusetzen. Neben dem Wunsch nach mehr gesetzlicher Regulation (Geschke et al. 2019) «schreit» das gewissermassen nach politischer Bildung online. Doch wie steht es um Hate Speech im Rahmen von Angeboten mit dem Anspruch politischer Bildung?

Neben vielfältigen digitalen Angeboten, die Hate Speech als Themenfeld politischer (Medien-)Bildung direkt adressieren (z.B. Die Modulbox «Politische Medienbildung für Jugendliche» im Auftrag des Deutschen Volkshochschul-Verbands in Kooperation mit dem Grimme-Institut ${ }^{10}$ ), können politische Bildungsangebote bzw. die darin handelnden Akteurinnen und Akteure sowie die Nutzerinnen und Nutzer solcher Angebote selbst zur Zielscheibe von Hassbotschaften werden.

Ernst et al. (2017) haben Nutzerinnen- und Nutzerkommentare $(n=155)$ unter acht Videos der Webvideoreihe «Begriffswelten Islam» qualitativ inhaltsanalysiert (vgl. Ernst et al. 2018, 30 f.). Dabei fanden die Forschenden heraus, dass sich viele Nutzerinnen und Nutzer abwertend gegenüber den Videoinhalten und anderen Nutzerinnen und Nutzern - inklusive des Moderations-Accounts der bpb - äussern.

Zahlreiche Studien aus der kommunikationswissenschaftlichen und medienpsychologischen Forschung legen nahe, dass die Art und Inhalte von Nutzerinnen- und Nutzerkommentaren die Wahrnehmung und Rezeption der Online-Angebote, auf die sie sich beziehen, beeinflussen können (z.B. Lee 2012; Weber 2014). Grundsätzlich wirken sich «unhöfliche» Nutzerinnen- und Nutzerkommentare negativ auf die Wahrnehmung der Textqualität aus und können Medien unglaubwürdig erscheinen lassen.

Natürlich hängt die Wirkung problematischer Medieninhalte (z.B. Hate Speech, extremistische Videos) von unterschiedlichen Voraussetzungen auf Seiten der Nutzerinnen und Nutzer, v.a. deren Persönlichkeitseigenschaften, Einstellungen, ihrem Vorwissen sowie individuellen Nutzungsbedingungen ab (Rieger et al. 2013; Schmitt

10 https://www.volkshochschule.de/verbandswelt/projekte/politische_jugendbildung/modulbox-zu-hatespeech-und-fake-news.php (Stand 14. Juli 2020). 
et al. 2017). Insbesondere gesellschaftliche Diskriminierungserfahrungen, die subjektive Wahrnehmung von Benachteiligung und Marginalisierung sowie positive Einstellungen gegenüber Macht, Gewalt und Aggression machen Menschen besonders empfänglich für derartige Inhalte (Miliopoulus 2017; Nordbruch 2016; Ribeau, Eisner, und Nivette 2017; Rieger et al. 2013).

Jedoch: Das Internet bzw. automatisierte Algorithmen und die Möglichkeit, aufgrund der unüberschaubaren Informations- und Meinungsvielfalt Informationen und Meinungen aus dem Weg zu gehen, die unserer persönlichen Haltung widersprechen, können Prozesse gesellschaftlicher Meinungspolarisierung bzw. die Entwicklung individueller problematischer Einstellungen begünstigen (Flaxman et al. 2016; Pariser 2011; Wojcieszak 2010). Fehlendes (Medien-)Wissen und eine mangelnde inhaltliche Auseinandersetzung mit Medieninhalten insbesondere bei jüngeren Mediennutzerinnen und -nutzern wirken sich zusätzlich ungünstig aus (z.B. Breem, Russel, und Weems 2001; Metzger, Flanagin, und Medders 2010; Shenton und Dixon 2003; Stanford History Education Group 2016).

Zwar berichten junge Mediennutzerinnen und -nutzer über ein «mulmiges» Gefühl beim Betrachten extremistischer Botschaften (Rieger et al., 2013), sie können jedoch das Gefühl bzw. dessen Ursachen nicht konkreter benennen. Ihnen fehlt zudem weitgehend die Kompetenz, Manipulationstechniken von Propaganda erkennen und reflektieren zu können - besonders deutlich wird dies in Bezug auf verschwörungserzählerische Inhalte (Craft, Ashley, und Maksl 2017; Schultz et al. 2017; Ernst et al. 2017). Diese Ergebnisse werden gestützt von zahlreichen weiteren Studien hinsichtlich der Medienkritikfähigkeit von Jugendlichen; sie erscheinen weitgehend unkritisch gegenüber der inhaltlichen Qualität von Informationen und ziehen selten die Möglichkeit in Betracht, dass andere als altruistische Motive des Autors ursächlich für die Mitteilung der gegebenen Informationen sein könnten. Eine breit angelegte Studie der Stanford History Education Group (2016) fand sogar heraus, dass jungen Mediennutzerinnen und -nutzern die Kompetenz zur Unterscheidung zwischen Nachrichten und sogenannten «Fake News» fehle. Sie lassen sich eher von optischen Heuristiken (z.B. professionelles Layout der Internetseite) leiten, anstatt sich gezielt mit medialen Inhalten auseinanderzusetzen (Metzger et al. 2010).

\section{Praxis politischer Bildung im Kontext digitaler Medien}

Vor dem Hintergrund charakteristischer Features, Strukturbedingungen digitaler, insbesondere aber auch sozialer Medien - algorithmisch generierter Empfehlungen und der Kommentarfunktion - soll nun abschliessend diskutiert werden, inwiefern politische Bildungsangebote im Netz ihren Zielsetzungen gerecht werden können. Die im Vorfeld ausgeführten Forschungsergebnisse machen deutlich: Politische Bildung allein in digitalen Medien birgt Risiken für Bildungsprozesse. 
Dies soll anhand unterschiedlicher Szenarien verdeutlicht werden. Dabei werden bewusst informelle mit formalen Rezeptions- bzw. Bildungssituationen verglichen, um vor diesem Hintergrund die Strukturbedingungen von YouTube didaktisch zu reflektieren.

\subsection{Worst-Case-Szenarien - Politische Bildung in digitalen Medien}

Zwei exemplarische Worst-Case-Szenarien sollen die Risiken digitaler Medien für Bildungsprozesse noch einmal verdeutlichen:

Szenario 1: Eine junge Rezipientin ruft ein Videoangebot der politischen Bildung zum Thema Islam auf YouTube ab. Nach etwa einer Minute erscheint ihr das Video etwas langatmig. Es wird ziemlich viel erklärt, einige Begriffe scheinen schwierig. Sie klickt auf ein in der Empfehlungsleiste vorgeschlagenes Videoangebot, in dem es ebenfalls um den Islam zu gehen scheint. Unsere junge Rezipientin gelangt ausgehend von einem Angebot der politischen Bildung wahlweise auf ein Angebot aus dem rechtsextremistischen oder islamistischen Spektrum.

Szenario 2: Ein junger Rezipient schaut sich auf YouTube ein Videoangebot der politischen Bildung zum Thema Islam an. Noch während des Schauens scrollt er hinunter zur Kommentarspalte und liest einige Kommentare. Er hinterlässt unter Videos sonst öfter Kommentare; positives Feedback, Rückfragen und Kritik an Aspekten, die ihm nicht zusagen. In der Kommentarspalte des abgerufenen Videoangebots heisst es an einigen Stellen, der Islam gehöre nicht zu Europa, geschweige denn zu Deutschland. Das sieht der junge Rezipient ganz anders. Doch anderen Nutzerinnen und Nutzer, die die Meinung des jungen Rezipienten teilen, werden Beleidigungen entgegengebracht, an einigen Stellen gar Drohungen gegen sie ausgesprochen. Der junge Rezipient entscheidet sich dazu, doch nicht am Diskurs zu partizipieren. Er erlebt Diskurse zu politischen Themen wie etwa «Islam in Deutschland» als hasserfüllt und bedrohlich.

Beide Szenarien werfen Fragen auf, die sich politische Bildung in digitalen Medien stellen muss: Wie werden die Inhalte eines Angebots rezipiert? Wird die Zielgruppe eines Angebots (angemessen) erreicht? Inwiefern beeinflussen die Rahmenbedingungen der Rezeption die Rezeption und die Verarbeitung der Inhalte?

Zwar liesse sich an dieser Stelle argumentieren, dass es eben auch darum geht, problematische Inhalte aus dem Internet zu entfernen. ${ }^{11}$ Wenngleich ein solcher bewahrender Schutz eine Unterstützung sein kann, bietet er jedoch auf der einen Seite nur einen kurzfristigen Schutz, da innerhalb kürzester Zeit neue Hasskommentare oder problematische Videos veröffentlicht werden. Oftmals bewegen sich bereits als problematisch einzustufende Inhalte auch unter der Schwelle der strafrechtlichen

11 Niederschlag gefunden hat dies beispielsweise im Netzwerkdurchsetzungsgesetz (NetzDG), welches im Oktober 2017 in Kraft getreten ist. 
Relevanz wie etwa Verschwörungserzählungen. Darüber hinaus stellen solche bewahrpädagogischen Ansätze nur eine Symptombehebung an der Oberfläche dar. Zudem kann das Löschen von Kommentaren und Inhalten für zahlreiche Nutzerinnen und Nutzer ein weiterer Beleg für vermeintliche Verschwörungen sein bzw. einen Anlass zur Elitenkritik darstellen.

In Bezug auf die beiden genannten Szenarien muss betont werden, dass diese überspitzte Worst-Case-Szenarien darstellen. Selbstredend müssen diese skizzierten Verläufe in dieser Form nicht eintreten. Ebenso vorstellbar sind nach Abruf eines politischen Bildungsangebots junge Rezipientinnen und Rezipienten, die auf der Grundlage der unterschiedlichen rezipierten Inhalte kritisch in neuen Kategorien reflektieren. Fraglos ist jedoch: Ohne didaktisches Handeln während des Rezeptionsprozesses von Online-Bildungsangeboten können sich die oben beschrieben Prozesse ereignen.

\subsection{Alternativszenarien - Politische Bildung mit digitalen Medien}

Eine Möglichkeit, der stärkeren Kontrolle der mit digitalen Medien einhergehenden Risiken kann lauten: Politische Bildung mit digitalen Medien. Angebote der politischen Bildung im Netz unter Begleitung politischer Bildnerinnen und Bildner bzw. Pädagoginnen und Pädagogen ist ein Ansatz, den skizzierten Risiken sinnvoll zu begegnen. Didaktik, didaktische Planung und didaktisches Handeln durch Pädagoginnen und Pädagogen, kann den beschrieben Risiken auf verschiedenen Ebenen entgegensteuern. Zwei Alternativszenarien politischer Bildung mit digitalen Medien sollen dies beispielhaft verdeutlichen:

Alternativszenario 1: Eine Lehrerin bereitet eine Unterrichtsreihe zum Thema Islamfeindlichkeit vor. Während der Vorbereitung stösst sie auf ein Angebot der Bundeszentrale für politische Bildung. Sie wählt ein thematisch passendes Video zum Einstieg in die Reihe aus. Im Unterricht präsentiert sie ihren Schülerinnen und Schülern das gesamte Video und moderiert eine anschliessende Plenumsdiskussion über die Videoinhalte mit ihren Schülerinnen und Schülern.

Alternativszenario 2: Ein Lehrer konzipiert eine Unterrichtsreihe zum Thema politische Meinungsbildung im Netz. Er wählt ein Videoangebot der Bundeszentrale für politische Bildung als Ausgangspunkt seiner Unterrichtsstunden, weil er in der Kommentarspalte dieser besonders spannende Diskussionen vermutet; schliesslich hat die bpb ja sogar mit einem eigenen Moderations-Account der Kommentare geworben! Er präsentiert seinen Schülerinnen und Schülern je einen konstruktiv-kritischen sowie einen hasserfüllten Kommentar von Nutzerinnen und Nutzern.

Alternativszenario 1 ähnelt zunächst dem bereits zu Beginn des Artikels skizzierten, «klassischen» Einsatz eines Videos als «Informationsträger» (Sander, 2005, 30). Zentral sind für sie die im Video übermittelten Informationen und Positionen, um 
die es auch in der Anschlusskommunikation gehen soll. In Alternativszenario 1 wird die hohe inhaltliche Qualität eines Bildungsangebots durch bewusstes Ausblenden etwaiger Nutzerinnen- und Nutzerkommentare mit problematischen Inhalten sowie weiterer Videos ausgespielt. Bei Bedarf bzw. in einer Fortsetzung einer solchen Sitzung kann die genannte Lehrerin etwa die Chancen und Risiken von sogenannten Empfehlungsalgorithmen und ihre potentielle Bedeutung für Meinungspolarisierung und das Entstehen von sogenannten Filterblasen und Echokammern thematisieren.

In Alternativszenario 2 wiederum wird das Bildungsangebot selbst zum Gegenstand. Der Lehrer verfolgt in seinem Unterricht ein anderes Lernziel als die erstgenannte Lehrerin und hat die Medienkompetenz bzw. zu initiierende (Medien-) Bildungsprozesse seiner Schülerinnen und Schüler im Blick. Mittels der Thematisierung von Nutzerinnen- und Nutzerkommentaren geht es nicht nur um Potenziale und Gefahren dieser im Netz, sondern auch um Grundfragen demokratischer Diskussionskultur und Hate Speech. In Alternativszenario 2 werden die potentiellen Einflussfaktoren auf die Rezeption des Angebots also bewusst in den Vordergrund gerückt - und so der potentielle Einfluss auf Rezipientinnen und Rezipienten pädagogisch aufgefangen bzw. aktiv thematisiert.

Die Vorgehensweisen beider Lehrpersonen fallen unter politische Bildung mit digitalen Medien. Gemeinsam ist beiden Szenarien die kompetente Reflexion potentieller Nebenwirkungen, Risiken und vor allem auch Chancen der Medienangebote für eine demokratische Gesellschaft.

\section{Implikationen für die Lehrpersonenbildung}

Was bedeutet das alles für eine medienpädagogisch informierte politische Bildung im Rahmen der Lehrpersonenbildung? Auf Grundlage der zuvor skizzierten Szenarien lassen sich einige Implikationen formulieren.

Als grundlegend ist anzusehen: Um Risiken und Chancen digitaler Medien einschätzen und didaktisch so verarbeiten zu können, dass Schülerinnen und Schüler in ihrer mündigen und selbstbestimmten Mediennutzung gefördert und Bildungsprozesse angestossen werden können, kommen Lehrpersonen nicht umhin, «teilnehmende Beobachter der medienkulturellen Welt ihrer Klientel» (Jörissen und Marotzki 2014, 329) zu werden oder in anderen Worten: Lehrpersonen müssen Bescheid wissen, was für ihre Schülerinnen und Schüler im Netz gerade eigentlich angesagt ist.

Hier ist das Ineinandergreifen politischer Bildung und Medienbildung bzw. Medienpädagogik auf Ebene der Lehrpersonenbildung gefragt: Denn politische Bildung mit digitalen Medien, die nicht ausschliesslich bewahrpädagogischen Grundsätzen folgt, setzt nicht nur das Vorhandensein von Angeboten voraus, sondern auch entsprechende Medienkompetenz bzw. «medienpädagogische Kompetenz» (Tulodziecki 
2012) aufseiten von Pädagoginnen und Pädagogen, ihre «didaktische Absicht» (Sander 2005, 30) erfolgreich in didaktisches Handeln überführen zu können.

Die Auswahl von Artikulationen in digitalen Medien für (medien-)pädagogische Situationen stellt spezifische Anforderungen an die handelnden Pädagoginnen und Pädagogen: Gefragt ist hier eine Form pädagogischer Medienkritik (Kübler 2006), d.h. eine «Medienkritik im Kontext pädagogischen Handelns» (Niesyto 2018, 59). Eine pädagogische Medienkritik digitaler Medien mit Blick (nicht nur) auf die politische Bildung darf nicht bei der Beurteilung medialer Artikulationen als «Werkzeuge» oder «Informationsträger» (Sander 2005, 30) stehenbleiben. Sie muss eben auch die Strukturbedingungen digitaler Medien kritisch betrachten und deren etwaigen (Neben-)Wirkungen in didaktischen Settings mitberücksichtigen. Konkret muss der Blick auf die algorithmisch generierten Empfehlungen weiterer Inhalte fallen: Auf welche "Klickpfade» schicke ich Jugendliche möglicherweise, wenn ich ihnen dieses oder jenes Video zeigen? Eingeschätzt werden muss auch der Einfluss von Nutzerinnen- und Nutzerkommentaren: Wie mögen Jugendliche ein Video möglicherweise (anders?) rezipieren, lesen sie vor, nach oder während der Rezeption die Nutzerinnen- und Nutzerkommentare?

Diese Fragen lassen sich freilich nicht rezeptartig beantworten und auch die zuvor skizzierten Szenarien sind notwendigerweise begrenzt. Bedeutsam ist jedoch: während politische Bildung in digitalen Medien hierauf kaum Einfluss zu nehmen vermag, so kann eine politische Bildung mit digitalen Medien hieran konkrete didaktische Entscheidungen anschliessen. Eine solche könnte etwa die Wahl darstellen, ein Video eingebettet, d.h. inklusive algorithmisch generierter Empfehlungen, Nutzerinnen- und Nutzerkommentare, Klicks, Titel etc., zu präsentieren oder auch isoliert, d.h. etwa im Vollbildmodus unter bewusster Ausblendung der zuvor aufgezählten Kontextfaktoren. Abhängig gemacht werden sollte eine solche Entscheidung letztlich von der Zielsetzung einer pädagogischen Situation - geht es mehr um den «Inhalt» eines Videos oder (auch?) um das Video als Video, als mediale Artikulation und Teil einer diskursiven, digitalen Welt?

Neben konkreten Kompetenzen sollten in diesem Zusammenhang grundsätzliche Unsicherheiten im Hinblick auf den Einsatz digitaler Medien im Unterricht nicht unterschätzt werden. In der Evaluation medienpädagogischer Unterrichtseinheiten von Schmitt et al. 2018 etwa äusserten sich Lehrpersonen besorgt, in ihrem Wissen zu und Kompetenzen im Gebrauch von digitalen Medien hinter ihren Schülerinnen und Schülern zurückzustehen. Hier müssen universitäre Lehrpersonenbildung und Angebote der Weiterbildung von bereits in der Praxis befindlichen Lehrpersonen ansetzen: Es braucht Räume für angehende und erfahrene Lehrpersonen, sich im Umgang mit digitalen Medien als selbstwirksam zu erfahren (für eine umfangreichere Diskussion siehe auch Schmitt et al. 2019). 


\section{6. $\quad$ Fazit}

Laut der bpb «initiiert und organisiert [politische] Bildungsprozesse, in denen es darum geht, unser individuelles Verhältnis zum Politischen zu bestimmen» (bpb, 2017b). Vor dem Hintergrund der zahlreichen Möglichkeiten digitaler Medien finden sich zunehmend mehr politische Bildungsangebote im Internet, zahlreiche Angebote binden dabei die vielfältigen Funktionen sozialer Medien mit ein, wie etwa die Möglichkeit, Medieninhalte in Kommentaren zu diskutieren.

Digitale Medien, insbesondere soziale Medien wie YouTube, bergen jedoch neben Chancen eine Vielzahl an Risiken - sowohl für ihre (jugendlichen) Nutzerinnen und Nutzer als auch für die Praxis politischer Bildung. Strukturbedingungen wie die auf YouTube thematisierten stellen die oben genannte (gezielte) Initiierung politischer Bildungsprozesse in digitalen Medien in Frage, da sie sich einer möglichen Einflussnahme entziehen.

Wird (gelingende) politische Bildung so aus der Hand gegeben? Angebote der politischen Bildung in digitale Medien zu verlegen, muss angesichts verschiedener (oben diskutierter) Faktoren, die erfolgreiche Bildungsprozesse zu stören vermögen, kritisch hinterfragt werden. Um potentielle Risiken zu minimieren und den Einfluss auf die Initiierung von Bildungsprozessen nicht preiszugeben, sollte die Reflexion der Besonderheiten digitaler Medien gegenüber analogen Medien zwingend notwendiger Bestandteil von Lernarrangements sein, die auf politische Bildungsprozesse abzielen. Je mehr die Medien durch Vereinnahmung immer weiterer Lebensbereiche an Bedeutung zunehmen, desto wichtiger wird die kritische pädagogische Auseinandersetzung mit deren Einflüssen auf menschliche Subjekte. Gleichsam geht es um die gezielte Auseinandersetzung mit den Chancen und Risiken digitaler Medien gemeinsam mit den Adressatinnen und Adressaten politischer Bildung (z.B. Jugendlichen). Pädagoginnen und Pädagogen brauchen hierzu Ressourcen wie Zeit, Geld und Mut für eine gezielte Weiterbildung mit dem Ziel des Schritthaltens mit aktuellen Entwicklungen digitaler Medien.

\section{Literatur}

Bakshy, Eytan, Solomon Messing, und Lada A. Adamic. 2015. «Exposure to ideologically diverse news and opinion on Facebook». Science, 348(6239): 1130-1132. https://doi.org/10.1126/ science.aaa1160.

Bettinger, Patrick 2018. Praxeologische Medienbildung. Theoretische und empirische Perspektiven auf sozio-mediale Habitustransformationen. Wiesbaden: Springer Fachmedien Wiesbaden. https://doi.org/10.1007/978-3-658-21849-2.

Brem, Sarah K., Janet Russel, und Lisa Weems. 2001. «Science on the web: Student evaluations of scientific arguments». Discourse Processes, 32(2-3): 191-213. https://doi.org/10.1080/0 163853X.2001.9651598. 
Bundeszentrale für politische Bildung (bpb). 2015. Begriffswelten Islam. http://www.bpb.de/ lernen/digitale-bildung/medienpaedagogik/213243/webvideos-begriffswelten-islam.

Bundeszentrale für politische Bildung (bpb). 2017a. \#erstewah/2017. http://www.bpb.de/politik/wahlen/bundestagswahl-2017/252400/erstewahl2017.

Bundeszentrale für politische Bildung (bpb). 2017b. Dossier Politische Bildung. http://www. bpb.de/gesellschaft/kultur/politische-bildung/.

Bundeszentrale für politische Bildung (bpb). 2019a. Say My Name. Webvideoprojekt. https://www.bpb.de/lernen/projekte/saymyname/?pk_campaign=nl2019-10-02\&pk_ $\mathrm{kwd}=293296$.

Bundeszentrale für politische Bildung (bpb). 2019b. Wahl-O-Mat zur Europawahl 2019. Interaktives Wahltool. https://www.bpb.de/politik/wahlen/wahl-o-mat/287903/europawahl-2019.

Busch, Andreas. 2017. «Informationsinflation: Herausforderungen an die politische Willensbildung in der digitalen Gesellschaft». In Medienkompetenz. Herausforderung für Politik, politische Bildung und Medienbildung, herausgegeben von Harald Gapski, Monika Oberle und Walter Staufer, 53-62. Bonn: Bundeszentrale für politische Bildung. https://www.bpb. de/system/files/dokument_pdf/10111_Medienkompetenz_ba.pdf.

Butler, Judith. 2016. Haß spricht. Zur Politik des Performativen. 5. Aufl. Berlin: Suhrkamp.

Calmbach, Mark, Silke Borgstedt, Inga Borchard, Peter Martin Thomas, und Berthold Bodo Flaig, Hrsg. 2016. Wie ticken Jugendliche 2016? Lebenswelten von Jugendlichen im Alter von 14 bis 17 Jahren in Deutschland. Wiesbaden: Springer. https://doi.org/10.1007/978-3-65812533-2.

Craft, Stephanie, Seth Ashley, und Adam Maksl. 2017. «News media literacy and conspiracyendorsement». Communication and the Public: 1-14. https://doi.org/10.1177/2057047317725539.

Eder, Sabine, Claudia Mikat, und Angela Tillmann, Hrsg. 2017. Software takes command. Herausforderungen der 'Datafizierung, für die Medienpädagogik in Theorie und Praxis. München: kopaed.

Ernst, Julian, Ann Kristin Beier, Josephine B. Schmitt, Diana Rieger, Sindyan Qasem, und HansJoachim Roth. 2017. « Muss doch nicht unbedingt mit Pistole sein, Alter.» - Lernarrangements zur Förderung von Medienkritikfähigkeit im Umgang mit extremistischer Internetpropaganda. Ein erster Evaluationsbericht aus dem Forschungsprojekt CONTRA». In Digitale Medien und politisch-weltanschaulicher Extremismus. Erkenntnisse aus Wissenschaft und Praxis, herausgegeben von Sally Hohnstein \& Maruta Herding, 189-212. Halle (Saale): Deutsches Jugendinstitut.

Ernst, Julian, Josephine B. Schmitt, Diana Rieger, Ann Kristin Beier, Peter Vorderer, Gary Bente, und Hans-Joachim Roth. 2017. «Hate beneath the counter speech? A qualitative content analysis of user comments on YouTube related to counter speech videos». Journal for Deradicalization (10): 1-49. https://journals.sfu.ca/jd/index.php/jd/article/view/91.

Flaxman, Seth, Sharad Goel, und Justin M. Rao. 2016. «Filter bubbles, echo chambers, and online news consumption». Public Opinion Quarterly, 80(S1), 298-320. https://doi. org/10.1093/poq/nfw006. 
Gapski, Harald, Monika Oberle, und Walter Staufer, Hrsg. 2017. Medienkompetenz. Herausforderung für Politik, politische Bildung und Medienbildung. Bonn: Bundeszentrale für politische Bildung. https://www.bpb.de/system/files/dokument_pdf/10111_Medienkompetenz_ba.pdf.

Geschke, Daniel, Anja Klaßen, Matthias Quent, und Christoph Richter. 2019. \#Hass im Netz: Der schleichende Angriff auf unsere Demokratie. https://www.idz-jena.de/forschungsprojekte/ hass-im-netz-der-schleichende-angriff-auf-unsere-demokratie-2018/.

handysektor. 2019. Fakt oder Fake: Das Handysektor Fake News Quiz. https://www.handysektor.de/artikel/fakt-oder-fake-das-handysektor-fake-news-quiz/.

Helberger, Natali, Kari Karppinen, und Lucia D’Acunto. 2016. «Exposure diversity as a design principle for recommender systems». Information, Communication \& Society: 1-17. https:// doi.org/10.1080/1369118X.2016.1271900.

Horkheimer, Max, und Theodor W. Adorno. 1969. Dialektik der Aufklärung. Philosophische Fragmente. Neuausgabe. Frankfurt am Main: Fischer.

Hugger, Kau-Uwe, und Lea Braun. 2015. «Videoclips im Internet als Identitätsressource von Jugendlichen». In Bewegte Bilder - Bewegende Pädagogik. Visuelle Medienkulturen in der Jugendmedienarbeit, herausgegeben von Jürgen Lauffer und Renate Röllecke, 19-24. München: kopaed.

Hugger, Kai-Uwe, und Ilona Cwielong. 2009. «Medienbildung als Umgang mit gesellschaftlicher Kontingenz in und mithilfe von Medien. Rezension von «Medienbildung - Eine Einführung)». MedienPädagogik, 101 (Reviews): 1-7. https://doi.org/10.21240/mpaed/XX/2009.12.30.X.

Hurrelmann, Klaus. 2012. «Jugendliche als produktive Realitätsverarbeiter: Zur Neuausgabe des Buches 〈Lebensphase Jugend»». Diskurs Kindheits- und Jugendforschung, 7, 89-100. https://www.budrich-journals.de/index.php/diskurs/article/view/6177.

Jörissen, Benjamin, und Winfried Marotzki. 2014. «Medienbildung in der digitalen Jugendkultur». In Digitale Jugendkulturen, Digitale Kultur und Kommunikation, herausgegeben von Kai-Uwe Hugger, 317-331. 2. Aufl. Wiesbaden: Springer Fachmedien. https://doi. org/10.1007/978-3-531-91908-9_7.

Jörissen, Benjamin, und Winfried Marotzki. 2009. Medienbildung - eine Einführung. Theorie Methoden - Analysen. Bad Heilbrunn: Klinkhardt.

Kenner, Steve, und Dirk Lange. 2020. «Bürgerbewusstsein, politisches Lernen und Partizipation im digitalen Zeitalter». DDS - Die Deutsche Schule, 112(2): 178-191. https://doi. org/10.31244/dds.2020.02.05.

Klinger, Ulrike, und Jakob Svensson. 2018. «The End of Media Logics? On Algorithms and Agency». New Media \& Society, 12. https://www.diva-portal.org/smash/get/diva2:1398862/FULLTEXT01.pdf.

Koller, Hans-Christoph. 2012. Bildung anders denken. Einführung in die Theorie transformatorischer Bildungsprozesse. Stuttgart: Kohlhammer.

Krotz, Friedrich. 2007. Mediatisierung. Fallstudien zum Wandel von Kommunikation. Wiesbaden: VS Verlag für Sozialwissenschaften. 
Kübler, Hans-Dieter. 2006. «Zurück zum «kritischen Rezipienten`? Aufgaben und Grenzen pädagogischer Medienkritik». In Medienkritik heute. Grundlagen, Beispiele und Praxisfelder, herausgegeben von Horst Niesyto, Matthias Rath, und Hubert Sowa, 17-52. München: kopaed.

Landesanstalt für Medien Nordrhein-Westfalen (LfM). 2016. Ethik im Netz. Hate Speech. http:// www.lfm-nrw.de/fileadmin/user_upload/Ifm-nrw/Service/Veranstaltungen_und_Preise/ Medienversammlung/2016/EthikimNetz_Hate_Speech-PP.pdf.

Lazer, David. 2015. «The rise of the social algorithm.» Science, 348(6239): 1090-1091. https:// doi.org/10.1126/science.aab1422.

Lee, Eun-Ju. 2012. «That's not the way it is: How user-generated comments on the news affect perceived media bias». Journal of Computer-Mediated Communication, 18(1): 32-45. https://doi.org/10.1111/j.1083-6101.2012.01597.x.

Leven, Ingo, und Hilde Utzmann. 2020. «Die Vielfalt der Digital Natives». In Jugend 2019. Eine Generation meldet sich zu Wort, herausgegeben von Shell Deutschland Holding, 247-287. Bonn: Bundeszentrale für politische Bildung.

Metzger, Miriam J., Andrew J. Flanagin, und Ryan B. Medders. 2010. «Social and heuristic approaches to credibility evaluation online». Journal of Communication, 60(3): 413-439. https://doi.org/10.1111/j.1460-2466.2010.01488.x.

Milbradt, Björn. 2018. Über Autoritäre Haltungen in spostfaktischen` Zeiten. Leverkusen-Opladen: Barbara Budrich-Esser.

Medienpädagogischer Forschungsverbund Südwest (mpfs). 2019. JIM-Studie 2018. Jugend, Information, Medien. Basisuntersuchung zum Medienumgang 12- bis 19-Jähriger. Stuttgart.

Baaken, Till, und Matthias Meyer. 2019. Die Peripherie des Extremismus auf YouTube. Die Blase. https://modus-zad.de/blog/die-blase-der-peripherie/.

Miliopoulus, Lazaros. 2017. «Biographische Verläufe im Extremismus: Ein kritischer Blick auf ihre Bedeutung für die Radikalisierungsforschung und die Extremismusprävention». In Politischer Extremismus im Vergleich, herausgegeben von Ralf Altenhof, Sarah Bunk, und Melanie Piepenschneider, 105-136. Berlin: LIT Verlag.

Moser, Heinz. 2011. «Das politische Internet - Möglichkeiten und Grenzen». MedienPädagogik, 21. https://doi.org/10.21240/mpaed/21/2011.11.08.X.

Niesyto, Horst. 2018. «Medienkritik - Entwicklungslinien und aktuelle Herausforderungen». In Medienkritik im digitalen Zeitalter, herausgegeben von Horst Niesyto und Heinz Moser, 5975. München: kopaed.

Nordbruch, Götz. 2016. The role of education in preventing radicalisation, RAN Issue Paper. http://www.ufuq.de/pdf/role_education_preventing_radicalisation.pdf.

Pariser, Eli. 2011. The Filter Bubble: What The Internet Is Hiding From You. London: Penguin UK.

Pöttinger, Ida, Tanja Kalwar, und Rüdiger Fries, Hrsg. 2016. Doing politics. Politisch agieren in der digitalen Gesellschaft. München: kopaed. 
Rat für Kulturelle Bildung. 2019. Jugend/YouTube/Kulturelle Bildung. Horizont 2019. Eine repräsentative Umfrage unter 12- bis 19-Jährigen zur Nutzung kultureller Bildungsangebote an digitalen Kulturorten. https://www.rat-kulturelle-bildung.de/fileadmin/user_upload/pdf/ Studie_YouTube_Webversion_final.pdf.

Reinemann, Carsten, Angela Nienierza, Nayla Fawzi, Claudia Riesmeyer, und Katharina Neumann. 2019. Jugend - Medien - Extremismus. Wo Jugendliche mit Extremismus in Kontakt kommen und wie sie ihn erkennen. Wiesbaden: Springer Fachmedien Wiesbaden. https:// doi.org/10.1007/978-3-658-23729-5.

Reheis, Fritz. 2016. Politische Bildung. Eine kritische Einführung. 2. Aufl. Wiesbaden: Springer VS. https://doi.org/10.1007/978-3-658-09463-8.

Ribeau, Denis, Manuel Eisner, und Amy Nivette. 2017. Können gewaltbereite extremistische Einstellungen vorausgesagt werden? http://www.media.uzh.ch/dam/jcr:41381576-3db24b9a-bb04-6464c538be16/Forschungsmemo.pdf.

Rieger, Diana, Lena Frischlich, und Gary Bente. 2013. Propaganda 2.0. Psychological Effects of Right-Wing and Islamic Extremist Internet Videos. Köln: Luchterhand Verlag.

Sander, Wolfgang. 2005. «Theorie der politischen Bildung: Geschichte - didaktische Konzeptionen - aktuelle Tendenzen und Probleme». In Handbuch politische Bildung, herausgegeben von Wolfgang Sander, 13-47. 3., völlig überarbeitete Auflage. Schwalbach im Taunus: Wochenschau Verlag.

Saurwein, Florian, Natascha Just, und Michael Latzer. 2015. «Governance of algorithms: options and limitations». Digital Policy, Regulation and Governance, 17(6): 35-49. https://doi. org/10.1108/info-05-2015-0025.

Schatto-Eckrodt, Tim, Svenja Boberg, Florian Wintterlin, Lena Frischlich, und Thorsten Quandt. 2019. «Bedrohte Deliberation. Information Warfare und Desinformation als Bedrohung digitaler Öffentlichkeiten». Communication Socialis, 52(2): 147-158. https://doi. org/10.5771/0010-3497-2019-2-147.

Schaumburg, Heike. 2011. "Politische Bildung und das Web 2.0». MedienPädagogik, 21. https://doi.org/10.21240/mpaed/21/2011.08.08.X.

Schmitt, Josephine B. 2014. "Onlinenachrichten und politisches Wissen bei Jugendlichen». Media Perspektiven, 1: 33-46.

Schmitt, Josephine B., Christina A. Debbelt, und Frank M. Schneider. 2017. «Too much information? Predictors of information overload in the context of online-news exposure». Information, Communication \& Society. https://doi.org/10.1080/1369118X.2017.1305427.

Schmitt, Josephine B., Julian Ernst, Lena Frischlich, und Diana Rieger. 2017. «Rechtsextreme und islamistische Propaganda im Internet: Methoden, Auswirkungen und Präventionsmöglichkeiten». In Politischer Extremismus im Vergleich, herausgegeben von Ralf Altenhof, Sarah Bunk, und Melanie Piepenschneider, 171-208. Berlin: LIT Verlag.

Schmitt, Josephine B., Diana Rieger, Olivia Rutkowski, und Julian Ernst. 2018. «Counter-messages as prevention or promotion of extremism?! The potential role of YouTube recommendation algorithms». Journal of Communication, 68(4): 780-808. https://doi.org/10.1093/ joc/jqy029. 
Schmitt, Josephine B., Diana Rieger, Julian Ernst, und Hans-Joachim Roth. 2018. «Critical media literacy and Islamist online propaganda: Feasibility, applicability and impact of three learning arrangements». International Journal of Conflict and Violence, 12. https://doi. $\operatorname{org} / 10.4119 /$ UNIBI/ijcv.642.

Schmitt, Josephine B., Claudia Riesmeyer, Julian Ernst, Diana Rieger, Angela Nienierza, Nayla Fawzi, Carsten Reinemann, und Hans-Joachim Roth. 2019. "Jugendliche und extremistische Propaganda. Kontakt - Schulische Prävention - Pädagogische Herausforderungen». Praxis der Rechtspsychologie, 1: 67-90.

Schultz, Tanjev, Nikolaus Jackob, Marc Ziegele, Oliver Quiring, und Christian Schemer. 2017. «Erosion des Vertrauens zwischen Medien und Publikum?». Media Perspektiven 5: 246-259. https://www.ard-werbung.de/fileadmin/user_upload/media-perspektiven/ pdf/2017/0517_Schultz_Jackob_Ziegele_Quiring_Schemer.pdf.

Shenton, Andrew K., und Pat Dixon. 2003. «Youngsters' use of other people as an informationseeking method». Journal of Librarianship and Information Science, 35(4): 219-233. https:// doi.org/10.1177/0961000603035004002.

Stalder, Felix. 2017. Kultur der Digitalität. 3. Auflage. Berlin: Suhrkamp.

Stanford History Education Group. 2016. Evaluating information: The cornerstone of civic online reasoning. https://sheg.stanford.edu/upload/V3LessonPlans/Executive\%20Summary\%20 11.21.16.pdf.

Statista. 2020. Statista-Dossier zu YouTube. https://de.statista.com/statistik/studie/id/12089/ dokument/youtube-statista-dossier/.

Süss, Daniel, und Eveline Hipeli. 2010. «Medien im Jugendalter». In Handbuch Mediensozialisation, herausgegeben von Ralf Vollbrecht und Claudia Wegener, 142-150. Wiesbaden: VSVerlag.

tagesschau. 2019. Der Mauerfall - digital erzählt. https://blog.tagesschau.de/2019/10/18/_ttrashed-7/.

Tarafdar, Monideepa, Christian Maier, Sven Laumer, und Tim Weitzel. 2019. «Explaning the link between technostress and technology addiction for social networking sites: A study of distraction as a coping behavior». information systems journal: 1-29. https://doi.org/10.1111/ isj. 12253.

Tulodziecki, Gerhard. 2012. «Medienpädagogische Kompetenz und Standards in der Lehrerbildung». In Jahrbuch Medienpädagogik 9 herausgegeben von Renate Schulz-Zander, Birgit Eickelmann, Heinz Moser, Horst Niesyto und Petra Grell. 271-297. Wiesbaden: VS Verlag für Sozialwissenschaften. https://doi.org/10.1007/978-3-531-94219-3_13.

Wachs, Sebastian. 2017. Gewalt im Netz. Studien über Risikofaktoren von Cyberbullying, Cybergrooming und Poly-Cyberviktimisierung unter Jugendlichen aus vier Ländern. Hamburg: Verlag Dr. Kovac.

Waldenfels, Bernhard. 2016. Topographie des Fremden. Studien zur Phänomenologie des Fremden 1. 7. Aufl. Frankfurt am Main: Suhrkamp. 
Wallace, Julian. 2018. «Modelling contemporary gatekeeping: the rise of individuals, algorithms and platforms in digital news dissemination». Digital Journalism, 6(3): 274-293. https://doi.org/10.1080/21670811.2017.1343648.

Weber, Patrick. 2014. "Discussions in the comments section: Factors influencing participation and interactivity in online newspapers' reader comments». New Media \& Society, 16(6): 941-957. https://doi.org/10.1177/1461444813495165.

Wojcieszak, Magdalena. 2010. «Don't talk to me»: effects of ideologically homogeneous online groups and politically dissimilar offline ties on extremism». New Media \& Society, 12(4): 637-655. https://doi.org/10.1177/1461444809342775.

Wolfert, Sabine, und Ingo Leven. 2020. «Freizeitgestaltung und Internetzugang: Wie Online und Offline ineinandergreifen». In Jugend 2019. Eine Generation meldet sich zu Wort herausgegeben von Shell Deutschland Holding, 213-242. Bonn: Sonderausgabe für die Bundeszentrale für politische Bildung.

Ziegele, Mark. 2016. Nutzerkommentare als Anschlusskommunikation. Theorie und qualitative Analyse des Diskussionswerts von Online-Nachrichten. Wiesbaden: Springer Fachmedien. https://doi.org/10.1007/978-3-658-12822-7.

Ziegele, Mark, Pablo Jost, Marike Bormann, und Dominique Heinbach. 2018. "Journalistic counter-voices in comment sections: Patterns, determinants, and potential consequences of interactive moderation of uncivil user comments». Studies in Communication and Media 4(7): 525-554. https://doi.org/10.5771/2192-4007-2018-4-525. 